\section{Reducing dimensionality effect in importance sampling simulations}

\section{J.L. Sanz-González, S. Zazo and F. Álvarez-Vaquero}

The dimensionality effect is avoided by the use of sufficient statistics in event probability estimators realised by importance sampling. If the system function is not a sufficient statistic, an approach is proposed to reduce the dimensionality effect in the estimators. Simulation results of false-alarm probability estimations, applied to radar detection, confirm a clear concordance with the theoretical results.

Introduction: As is well-known, the importance sampling (IS) technique [1-4] is a modified Monte-Carlo simulation applied to rare-event probability estimation, such as estimation of very low false-alarm probability in radar detection, or very low error-probability in communications.

Consider a random vector $\boldsymbol{X}=\left(X_{1}, X_{2}, \ldots, X_{n}\right)$ and its probability density function (PDF) $f_{\boldsymbol{X}}(\boldsymbol{x})$ defined in the $R^{n}$-space, being $\boldsymbol{x}=\left(x_{1}\right.$, $\left.x_{2}, \ldots, x_{n}\right)$ a vector of real components. Define a statistic $t(\boldsymbol{x})$ and the critical region $t(\boldsymbol{x}) \geq t_{0}$ in the $R^{n}$-space; the probability $P_{0}=\operatorname{Pr}\{t(\boldsymbol{X}) \geq$ $\left.t_{0}\right\}=E\left\{u\left(t(\boldsymbol{X})-t_{0}\right)\right\}$, where $E\{\cdot\}$ means expectation with respect to $f_{\boldsymbol{X}}(\boldsymbol{x})$ and $u(\cdot)$ is the unit-step function. An alternative equation for this probability is $P_{0}=E^{*}\left\{w_{\boldsymbol{X}}(\boldsymbol{X}) \cdot u\left(t(\boldsymbol{X})-t_{0}\right)\right\}$, where $E^{*}\{\cdot\}$ means expectation with respect to $f_{X}^{*}(x)$, known as the IS-PDF or the biasing PDF, and $w_{X}(x)=f_{X}(x) / f_{X}^{*}(x)$ is the weighting function. An estimator of $P_{0}$ is the sample mean $P_{0}$, i.e.

$$
\hat{P}_{0}=\frac{1}{N} \sum_{k=1}^{N} w_{\boldsymbol{X}}\left(\boldsymbol{x}_{k}^{*}\right) \cdot u\left(t\left(\boldsymbol{x}_{k}^{*}\right)-t_{0}\right)
$$

being $\boldsymbol{x}_{k}^{*}$ and $k=1,2, \ldots, N$, independent sample vectors with PDF $f_{X}^{*}(\boldsymbol{x})$.

If $f_{X}^{*}(x) \neq 0$ wherever $f_{\boldsymbol{X}}(\boldsymbol{x}) \neq 0$ in $t(\boldsymbol{x}) \geq t_{0}, \boldsymbol{x} \in R^{n}$, the estimator $\hat{P}_{0}$ is unbiased and consistent [1]. In fact, the variance $\sigma_{\hat{P}_{0}}^{2}$ of $\hat{P}_{0}$ is

$$
\sigma_{\hat{P}_{0}}^{2}=E^{*}\left\{\left(\hat{P}_{0}-P_{0}\right)^{2}\right\}=\frac{1}{N}\left(E^{*}\left\{\left[w_{\boldsymbol{X}}(\boldsymbol{X}) \cdot u\left(t(\boldsymbol{X})-t_{0}\right)\right]^{2}\right\}-P_{0}^{2}\right)
$$

As is well-known [1], $f_{\boldsymbol{X}}^{*}(\boldsymbol{x})=f_{\boldsymbol{X}}(\boldsymbol{x}) \cdot u\left(t(\boldsymbol{x})-t_{0}\right) / P_{0}$ provides zero variance in (2) for any $N \geq 1$; however, it is not practical because $P_{0}$ is unknown (in fact, it has to be estimated). In the literature [1-4], some families of $f_{X}^{*}(\boldsymbol{x})$ have been proposed for different estimation problems and the optimal solution is constrained to this family.

An important problem in many IS simulations is the dimensionality effect [2], meaning that $\sigma_{\hat{P}_{0}}^{2}$ increases as the dimension $n$ of vector $\boldsymbol{x}$ increases, for a constant number $N$ of simulation runs.

Reducing the dimensionality effect: If $P_{0}$ and $N$ are fixed in (2), to minimise $E^{*}\left\{\left[w_{\boldsymbol{X}}(\boldsymbol{X}) \cdot u\left(t(\boldsymbol{X})-t_{0}\right)\right]^{2}\right\}$ is equivalent to minimising $\sigma_{\hat{P}_{0}}^{2}$

Proposition 1. Define the weighting finctions: $w_{X}(x)=f_{X}(x) / f_{X}^{*}(x)$ and $w_{T}(t)=f_{T}(t) / f_{T}^{*}(t)$, where $t=t(\boldsymbol{x})$ and the random variable $T=t(\boldsymbol{X})$ with $f_{T}(\cdot)$ and $f_{T}^{*}(\cdot)$ the corresponding induced PDF's; then, the following inequality holds:

$$
E^{*}\left\{\left[w_{\boldsymbol{X}}(\boldsymbol{X}) \cdot u\left(t(\boldsymbol{X})-t_{0}\right)\right]^{2}\right\} \geq E^{*}\left\{\left[w_{T}(T) \cdot u\left(T-t_{0}\right)\right]^{2}\right\}
$$

Proof: Using conditional expectation, we can write

$$
E^{*}\left\{\left[w_{\boldsymbol{X}}(\boldsymbol{X}) \cdot u\left(t(\boldsymbol{X})-t_{0}\right)\right]^{2}\right\}=E^{*}\left\{E^{*}\left\{\left[w_{\boldsymbol{X}}(\boldsymbol{X}) \cdot u\left(t(\boldsymbol{X})-t_{0}\right)\right]^{2} \mid T\right\}\right\}
$$

Considering that the variance is a non-negative number (or by Jensen's inequality), we can establish the following inequality:

$$
E^{*}\left\{\left[w_{\boldsymbol{X}}(\boldsymbol{X}) \cdot u\left(t(\boldsymbol{X})-t_{0}\right)\right]^{2} \mid t\right\} \geq\left[E^{*}\left\{w_{\boldsymbol{X}}(\boldsymbol{X}) \cdot u\left(t(\boldsymbol{X})-t_{0}\right) \mid t\right\}\right]^{2}
$$

Also, $E^{*}\left\{w_{\boldsymbol{X}}(\boldsymbol{X}) u\left(t(\boldsymbol{X})-t_{0}\right) \mid t\right\}=\int_{R^{n}} w_{\boldsymbol{X}}(\boldsymbol{x}) u\left(t(\boldsymbol{x})-t_{0}\right) f_{\boldsymbol{X} \mid T}^{*}(\boldsymbol{x} \mid t) \mathrm{d} \boldsymbol{x}$ and $f_{X \mid T}^{*}(x \mid t)=f_{X}^{*}(x) f_{T \mid X}^{*}(t \mid x) / f_{T}^{*}(t), f_{T \mid X}^{*}(t \mid x)=f_{T \mid X}(t \mid x)=\delta(t-t(x)), \delta(\cdot)$ being the Dirac delta function. Finally, after substituting the above, thus we have $E^{*}\left\{w_{\boldsymbol{X}}(\boldsymbol{X}) \cdot u\left(t(\boldsymbol{X})-t_{0}\right) / t\right\}=w_{T}(t) \cdot u\left(t-t_{0}\right)$, and Proposition 1 has been proved.
Proposition 2: If $t(\boldsymbol{x})$ is a sufficient statistic for the family of $f_{X}^{*}(\boldsymbol{x})$ and $f_{\boldsymbol{X}}(\boldsymbol{x})$, then

$$
E^{*}\left\{\left[w_{\boldsymbol{X}}(\boldsymbol{X}) \cdot u\left(t(\boldsymbol{X})-t_{0}\right)\right]^{2}\right\}=E^{*}\left\{\left[w_{T}(T) \cdot u\left(T-t_{0}\right)\right]^{2}\right\}
$$

Proof: Owing to the property of sufficiency: $f_{X}^{*}(x)=g(x) \cdot h^{*}(t(x))$ and $f_{\boldsymbol{X}}(\boldsymbol{x})=g(\boldsymbol{x}) \cdot h(t(\boldsymbol{x}))$, we have

$$
E^{*}\left\{\left[w_{\boldsymbol{X}}(\boldsymbol{X}) \cdot u\left(t(\boldsymbol{X})-t_{0}\right)\right]^{2} \mid t\right\}=\left[h(t) / h^{*}(t) \cdot u\left(t-t_{0}\right)\right]^{2}
$$

From the PDF definition (assuming $\mathrm{d} t>0$ and $\mathrm{d} t \rightarrow 0$ )

$$
\begin{aligned}
f_{T}(t) \mathrm{d} t & =\operatorname{Pr}\{t \leq t(\boldsymbol{X})<t+\mathrm{d} t\}=\int_{t \leq t(x)<t+\mathrm{d} t} f_{X}(\boldsymbol{x}) \mathrm{d} \boldsymbol{x} \\
& =h(t) \int_{t \leq t(x)<t+\mathrm{d} t} g(\boldsymbol{x}) \mathrm{d} \boldsymbol{x}
\end{aligned}
$$

and

$$
f_{T}^{*}(t) \mathrm{d} t=h^{*}(t) \int_{t \leq t(x)<t+\mathrm{d} t} g(\boldsymbol{x}) \mathrm{d} \boldsymbol{x}
$$

we can write $w_{T}(t)=f_{T}(t) / f_{T}^{*}(t)=h(t) / h^{*}(t)$ and its substitution in (5) finishes the proof.

Proposition 1 means that the variance of $\hat{P}_{0}$ realised by $w_{\boldsymbol{X}}(\boldsymbol{x})$ in (1) is greater than the variance of $\hat{P}_{0}$ realised by $w_{T}(t)$ in place of $w_{\boldsymbol{X}}(\boldsymbol{x})$, unless $t(\boldsymbol{x})$ is a sufficient statistic according to Proposition 2, where the dimensionality effect is cancelled.

Finally, if $t(x)$ is not a sufficient statistic, we should compute or estimate the weighting function $w_{T}(t)$, in order to be used in place of $w_{\boldsymbol{X}}(\boldsymbol{x})$ in (1) for reducing the dimensionality effect in the estimation of $P_{0}$. The computation of $w_{T}(t)$ should be performed through the computation of $f_{T}(\cdot)$ and $f_{T}^{*}(\cdot)$ over $t=t(\boldsymbol{x})$ from $f_{\boldsymbol{X}}(\boldsymbol{x})$ and $f_{X}^{*}(\boldsymbol{x})$, respectively. This subject is now under research by the authors of this Letter.

Applications to radar detection: Consider the binary hypotheses $H_{0}$ (target absent) and $H_{1}$ (target present), defined as follows: $H_{0}: f_{\boldsymbol{X}}\left(\boldsymbol{x} \mid H_{0}\right)=\prod_{i=1}^{n} f_{0}\left(x_{i}\right)$ and $H_{1}: f_{\boldsymbol{X}}\left(\boldsymbol{x} \mid H_{1}\right)=\prod_{i=1}^{n} f_{1}\left(x_{i}\right)$, where $\boldsymbol{x}=\left(x_{1}\right.$, $x_{2}, \ldots, x_{n}$ ) is the vector of $n$ independent envelope samples ( $n$ is the number of pulses per antenna beam width), $f_{0}(x)$ is the PDF of the noise sample and $f_{1}(x)$ is the PDF of the target signal with noise. For the Gaussian noise, $f_{0}(x)$ is the Rayleigh distribution; for a nonfluctuating (NF) target model, $f_{1}(x)$ is the Rice distribution with $S$ as the signal-to-noise ratio parameter; for the Swerling II (SW-II) target model [5], $f_{1}(x)$ is the Rayleigh distribution with parameter $S_{0}$ the signal-to-noise ratio mean. The optimum Neyman-Pearson detector has the well-known structure [5]

$$
t(\boldsymbol{x})=\sum_{i=1}^{n} a\left(x_{i}\right) \stackrel{H_{1}}{<} t_{0}
$$

where $a(x) \simeq x$ (linear) for a NF target model, and $a(x)=x^{2}$ (quadratic) for the SW-II target model. The false-alarm probability $\left(P_{\text {fa }}\right)$ is defined by $P_{\mathrm{fa}}=\operatorname{Pr}\left\{t(\boldsymbol{X}) \geq t_{0} / H_{0}\right\}$, hence, we can identify $P_{\mathrm{fa}}$ with $P_{0}$ and $f_{\boldsymbol{X}}\left(\boldsymbol{x} / H_{0}\right)$ with $f_{\boldsymbol{X}}(\boldsymbol{x})$ given above. According to Orsak [3], a good IS biasing PDF is $f_{\boldsymbol{X}}^{*}(\boldsymbol{x})=f_{\boldsymbol{X}}\left(\boldsymbol{x} \mid H_{1}\right)$ with an adequate signal-to-noise ratio. Since the linear statistic is a sufficient statistic for detecting a NF target model, the adequate IS biasing PDF should correspond to the NF case, in order to cancel the dimensionality effect; on the other hand, quadratic statistic is a sufficient statistic for the SW-II target model, and the adequate IS biasing PDF should correspond to the SW-II case for cancelling the dimensionality effect.

Simulation results: Now, we consider the linear and quadratic statistics as detector statistics in (6), and estimate the false-alarm probability by using IS PDF's corresponding to the NF and SW-II target models. The results are shown in Figs. 1 and 2, where the parameter values are: number of integrated pulses, $n=8$; number of simulation runs, $N=1000$; IS-biasing parameters, $S=S_{0}=3$. In Fig. 1 , we show

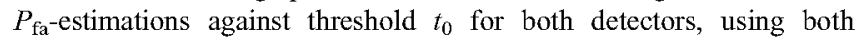
biasing PDF's: NF (NF: dotted lines) and SW-II (SW-II: solid lines); in addition, we show theoretical curves (discontinuous red lines) of 
$P_{\mathrm{fa}}$-values (computed from integral equations). In Fig. 2, we show the corresponding relative-error estimation ( $\hat{\varepsilon})$ of $P_{\mathrm{fa}}$-estimations:

$$
\hat{\varepsilon}=\sqrt{\hat{\sigma}_{\hat{P}_{\mathrm{fa}}}^{2}} / \hat{P}_{\mathrm{fa}}
$$

for the four possibilities, where $\hat{\sigma}_{\hat{P}_{\text {f }}}^{2}$ is a variance estimator of $\hat{P}_{\mathrm{fa}}$. From Figs. 1 and 2, it can be seen that for the quadratic detector, $\hat{P}_{\mathrm{fa}}$-values obtained by SW-II as IS-PDF are very close to the exact $P_{\mathrm{fa}}$-values, and better than those obtained by the NF procedure. On the contrary, for the linear detector, the $\hat{P}_{\mathrm{fa}}$ provided by a NF as IS-PDF is better than that provided by the SW-II procedure. Similar results are obtained for $n=16,32$ or 64 .

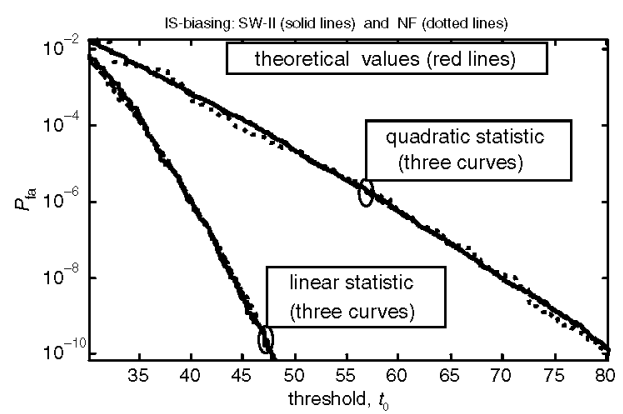

Fig. 1 False-alarm probability $\left(P_{f a}\right)$ against threshold to for linear and quadratic detectors: theoretical values and estimations by two different IS-PDF"s (SW-II and NF)

Parameters: $n=8, S=S_{0}=3$ and $N=1000$

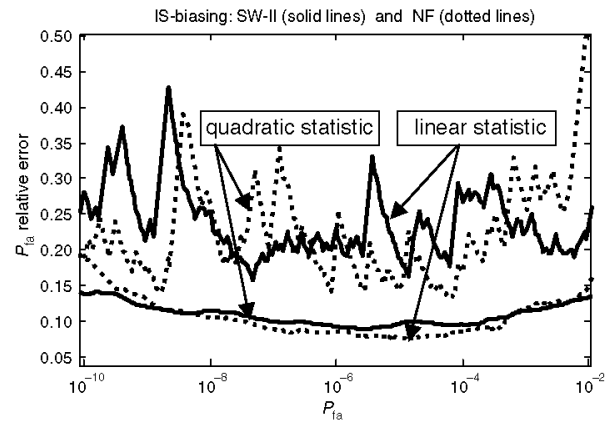

Fig. $2 P_{f a}$-relative error ( $\hat{\varepsilon}$ ) against $P_{f a}$ in same conditions of Fig. 1
Conclusion: It has been shown that if the system statistic is sufficient, the dimensionality effect is circumvented in rare-event probability estimation by the IS technique. Applications to false-alarm probability estimations in radar detectors have been given, and the simulation results are in agreement with the theoretical ones.

Acknowledgements: This work was supported by the Ministerio de Ciencia e Innovación (grant TEC2010-21217-C02-02/TCM), and the 'Universidad Politécnica de Madrid.

(C) The Institution of Engineering and Technology 2013

26 April 2013

doi: $10.1049 / \mathrm{el} .2013 .1451$

One or more of the Figures in this Letter are available in colour online. J.L. Sanz-González, S. Zazo and F. Álvarez-Vaquero (Dpto. SSR, Universidad Politécnica de Madrid, ETSI de Telecomunicación-UPM, Ciudad Universitaria, Madrid 28040, Spain)

E-mail: jlsanz@gcs.ssr.upm.es

\section{References}

1 Chen, J.C., Lu, D., Sadowsky, J.S., and Yao, K.: 'On importance sampling in digital communications - Part I: Fundamentals', IEEE J. Sel. Areas Commun., 1993, 11, (3), pp. 289-299

2 Smith, P.J., Shaf, M., and Gao, H.: 'Quick simulation: a review of importance sampling techniques in communications systems', IEEE J. Sel. Areas Commun., 1997, 15, (4), pp. 597-613

3 Orsak, G.C.: 'A note on estimating false alarm rates via importance sampling', IEEE Trans. Commun., 1993, 41, (9), pp. 1275-1277

4 Vicen-Bueno, R., Jarabo-Amores, M.P., Rosa-Zurera, M., Sanz-González, J.L., and Maldonado-Bascón, S.: 'Importance sampling for objective function estimations in neural detector training driven by genetic algorithms', Neural Process. Lett., 2010, 32, (3), pp. 249-268

5 Peebles, P.Z.: 'Radar principles' (John Wiley \& Sons, Inc., New York, 1998) 